\title{
LINEAR VISCOELASTIC ACTUATOR-BASED CONTROL SYSTEM OF A BIPEDAL WALKING ROBOT
}

\author{
V. Berbyuk* ${ }^{*}$ B. Peterson ${ }^{* *}$ and N. Nishchenko* \\ *Pidstryhach Institute for Applied Problems of Mechanics and Mathematics \\ Ukrainian National Academy of Sciences \\ 3-B, Naukova St., 290601, Lviv, Ukraine \\ e-mail: berbyuk@iapmm.lviv.ua \\ ** Department of Mechanics, Chalmers University of Technology \\ S-412 96, Gothenburg, Sweden \\ e-mail: bope@mec.chalmers.se
}

\begin{abstract}
The optimisation approach for designing of the rotational spring-damper actuators providing the programmed goal-directed motion of a bipedal walking robot is proposed. The problem is formulated as an approximation procedure for the controlling torques acting at the joints of the robot during its optimal motion. Analysis of the obtained numerical results has shown that the anthropomorphic energy-optimal goal-directed motion of the bipedal walking robot could be generated by the rotational spring-damper actuators with one switching of each of their parameters during the double step of the robot.
\end{abstract}

\section{INTRODUCTION}

The complexity of the structure and the dynamical instability of the gait of the bipedal walking robots (BWR) make it very difficult to understand the main features of their control systems needed to provide the goal-directed stable motion. A number of mathematical models of the bipedal locomotion systems with different degrees of -complexity have been proposed during last two decades [1-11]. These models were used for different purposes, e.g. to design the anthropomorphic mechatronic system (exoskeleton) [1], to study human normal gait $[3,8]$ and pathological gait $[8,9]$, to develop the control systems for the BWR [1-7], etc.
Nevertheless, presently there is not enough information about how to create an effective control system of the BWR, especially to provide its extremal, e.g. time-optimal or energyoptimal motion. What type of controlling forces of the actuators are required for these extremal controlled motion of the robots?

The central idea of this paper is that an effective control system of a mobile robot can be designed based on a combination of passive and active controlling stimuli. The passive actuators have to provide the programmed goal-directed motion of the robot. The active controlling stimuli should be used for some other aims, e.g. to solve the stabilisation problem of motion of the robot. 
The optimisation approach for designing of the rotational springdamper actuators providing the programmed goal-directed motion of a bipedal walking robot is proposed. The problem is formulated as the approximation procedure of the controlling torques acting at the joints of the robot during its optimal motion. This motion and respective torques are determined by solution of the optimal control problem for the dynamical system modelling the robot. In the optimal control problem the objective function has been chosen as the integral over a double step of the sum of the absolute values of the mechanical power of all controlling torques acting at the joints of the robot [5-7]. The restrictions on the phase coordinates and the controlling forces have been generated based on experimental data of human locomotion [8]. In order to solve the nonlinear optimal control problem an approach based on the inverse dynamics method and the Rosenbrock's algorithm has been used [10].

Analysis of the obtained numerical results has shown that the anthropomorphic energy-optimal goal-directed motion of the bipedal walking robot could be generated by the rotational spring-damper actuators with one switching for each of their parameters during the double step of the robot.

\section{MATHEMATICAL MODEL}

The BWR under consideration consists of an inertial torso with weight and two identical legs (Fig.1). Each leg consists of three elements. The two elements with mass and rotatory inertia model the thigh and the shank, while a third massless and inertia-free element models the foot.

Let the system OXYZ be a fixed rectangular Cartesian coordinate system. It is assumed that the robot moves in the OXY plane along the OX axis, over a horizontal surface.

In addition to the weight of the torso, thighs and shanks, the external forces acting on the BWR include the interaction forces between the feet and the surface, which are replaced by the force $\mathbf{R}_{i}(t)$ applied at the point of the ankle joint $\mathrm{Ai}$, and the moment $p_{i}(t)$ that acts in the same joint $(i=1,2)$. The force $\mathbf{R}_{i}(t)$ is the principal vector of the plane system of reaction forces of the support; $p_{i}(t)$ is the principal moment of the reaction forces of the support, referred to the point of the ankle joint. It is also assumed that the control torques $q_{i}(t)$ and $u_{i}(t)(i=1,2)$ are acting at the hip $\mathrm{H}$ and knee $\mathrm{Ki}$ joints, respectively.

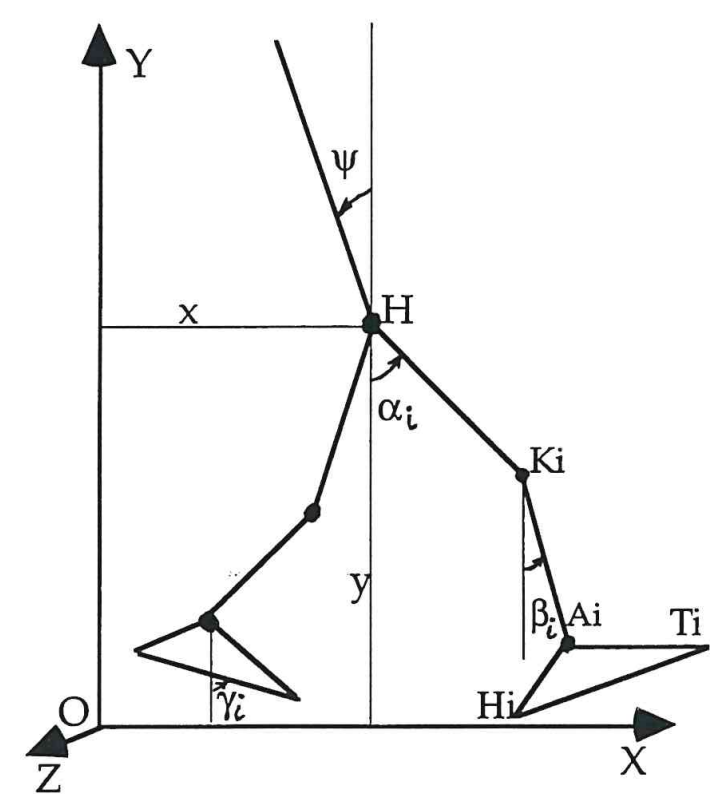

Fig.1 Model of the Bipedal Walking Robot

The controlled motion of the BWR can be described by the Lagrange's equations of the second 
kind and the kineto-static balance conditions for the feet under the action of the ankle moment and the reaction of the support $[7,9,10]$ :

$$
\begin{aligned}
& f_{1}-K_{r}\left(\ddot{\psi} \cos \psi-\dot{\psi}^{2} \sin \psi\right)=R_{1 x}+R_{2 x} \\
& f_{2}-K_{r}\left(\ddot{\psi} \sin \psi+\dot{\psi}^{2} \cos \psi\right)=R_{1 y}+R_{2 y} \\
& f_{3 i}=q_{i}-u_{i}+a\left(R_{i x} \cos \alpha_{i}+R_{i y} \sin \alpha_{i}\right) \\
& f_{4 i}=u_{i}-p_{i}+b\left(R_{i x} \cos \beta_{i}+R_{i y} \sin \beta_{i}\right) \\
& f_{5}=-q_{1}-q_{2} \\
& p_{i}+\left(y_{i}-y_{R i}\right) R_{i x}-\left(x_{i}-x_{R i}\right) R_{i y}=0
\end{aligned}
$$

where

$$
\begin{aligned}
& f_{1}=M \ddot{x}+\sum_{i=1}^{2}\left[K_{a}\left(\dot{\alpha}_{i} \cos \alpha_{i}\right)^{\cdot}+K_{b}\left(\dot{\beta}_{i} \cos \beta_{i}\right)^{\cdot}\right] \\
& f_{2}=M(\ddot{y}+g)+\sum_{i=1}^{2}\left[K_{a}\left(\dot{\alpha}_{i} \sin \alpha_{i}\right)^{\cdot}+\right. \\
& \left.K_{b}\left(\dot{\beta}_{i} \sin \beta_{i}\right)^{\cdot}\right] \\
& f_{3 i}=J_{1} \ddot{\alpha}_{i}+K_{a}\left(\ddot{x} \cos \alpha_{i}+\ddot{y} \sin \alpha_{i}\right)+ \\
& a K_{b}\left[\ddot{\beta}_{i} \cos \left(\alpha_{i}-\beta_{i}\right)+\dot{\beta}_{i}^{2} \sin \left(\alpha_{i}-\beta_{i}\right)\right]+ \\
& g K_{a} \sin \alpha_{i}, \quad(i=1,2) \\
& f_{4 i}=J_{b} \ddot{\beta}_{i}+K_{b}\left(\ddot{x} \cos \beta_{i}+\ddot{y} \sin \beta_{i}\right)+ \\
& a K_{b}\left[\ddot{\alpha} \cos \left(\alpha_{i}-\beta_{i}\right)-\dot{\alpha}_{i}^{2} \sin \left(\alpha_{i}-\beta_{i}\right)\right]+ \\
& g K_{b} \sin \beta_{i}, \quad(i=1,2) \\
& f_{5}=J \ddot{\psi}-K_{r}(\ddot{x} \cos \psi+\ddot{y} \sin \psi)-g K_{r} \sin \psi \\
& M=m+2 m_{a}+2 m_{b}, \quad K_{a}=m_{a} r_{a}+a m_{b} \\
& K_{b}=m_{b} r_{b}, \quad K_{r}=r m, \quad J_{1}=J_{a}+a^{2} m_{b}
\end{aligned}
$$

In the above expressions: $x, y, \psi, \alpha_{i}, \beta_{i}, \gamma_{i}$ are the Cartesian coordinates of the suspension point $\mathrm{H}$ of the legs and the angles that specify the position of the elements of the BWR (Fig.1); $m, r, J$ are the mass, the distance from the suspension point of the legs to the centre of mass and the moment of inertia of the torso relative to the $\mathrm{Z}$ axis at point $\mathrm{H}$, respectively; $m_{a}, r_{a}, a, J_{a}$ are the mass, the distance from $\mathrm{H}$ to the centre of mass, the length and the moment of inertia of the thigh relative to the $\mathrm{Z}$ axis at $\mathrm{H}$, respectively; $m_{b}, r_{b}, b, J_{b}$ are the mass, the distance from $\mathrm{Ki}$ to the centre of mass, the length and the moment of inertia of the shank relative to the $Z$ axis at the point $K i$, respectively; $R_{i x}, R_{i y}$ are the horizontal and vertical components of the force $\mathbf{R}_{i}(t) ;\left(x_{i}, y_{i}\right),\left(x_{R i}, y_{R i}\right)$ are the Cartesian coordinates of the ankle joint and of the point of application of the vector $\mathbf{R}_{i}(t)$ of the i-th leg, respectively; $g$ is the acceleration due to gravity, and the dot " $"$ is a derivative with respect to time.

\section{STATEMENT OF THE PROBLEM}

The object under consideration is a nonlinear multidimensional controlled dynamical system. The problem of designing the passive actuators is formulated as the approximation procedure of the controlling torques acting at the joints of the robot during its energy-optimal motion.

Let us describe some kinematic characteristics of the typical gait of the BWR before stating of the respective optimal control problem.

We shall assume that there are four phases of the leg action during a double step of the robot $(t \in[0, T])$ : the first double $\left(t \in\left[0, t_{1}\right]\right)$ and single $\left(t \in\left[t_{1}, t_{2}\right]\right)$ support phases, and the second double $\left(t \in\left[t_{2}, t_{3}\right]\right)$ and single $\left(t \in\left[t_{3}, T\right]\right)$ support phases [6-8]. This rhythm of the double step of the robot leads to the following kinematic constraints:

$$
\begin{aligned}
& x_{H 1}(t)=x_{H 1}^{0}, \quad y_{H 1}(t)=0, \quad t \in\left[0, t_{1}\right] \\
& x_{T 2}(t)=x_{T 2}^{0}, \quad y_{T 2}(t)=0, \quad t \in\left[0, t_{1}\right] \quad(2) \\
& x_{T 1}(t)=x_{H 1}^{0}+l, \quad y_{T 1}(t)=0, \quad t \in\left[t_{1}, t_{3}\right] \\
& x_{H 2}(t)=x_{H 1}^{0}+L, \quad y_{H 2}(t)=0, \quad t \in\left[t_{2}, t_{3}\right] \\
& x_{T 2}(t)=x_{T 2}^{0}+2 L, \quad y_{T 2}(t)=0, \quad t \in\left[t_{3}, T\right] \\
& x_{H 1}(T)=x_{H 1}^{0}+2 L, \quad y_{H 1}(T)=0,
\end{aligned}
$$


where $x_{H 1}^{0}, x_{T 2}^{0}, t_{1}, t_{2}, t_{3}, T$ are given parameters; $l$ is the length of the foot, $L$ is the length of the single step; $\left(x_{H i}, y_{H i}\right),\left(x_{T i}, y_{T i}\right)$ are the Cartesian coordinates of the heel and toe of the i-th leg, respectively. These coordinates are determined by the expressions (Fig.1):

$$
\begin{aligned}
& x_{H i}(t)=x_{i}-l_{1} \cos \left(\gamma_{i}-\varphi\right) \\
& y_{H i}(t)=y_{i}-l_{1} \sin \left(\gamma_{i}-\varphi\right) \\
& x_{T i}(t)=x_{i}+l_{2} \sin \left(\gamma_{i}-\varphi\right) \\
& y_{T i}(t)=y_{i}-l_{2} \cos \left(\gamma_{i}-\varphi\right) \\
& x_{i}(t)=x+a \sin \alpha_{i}+b \sin \beta_{i} \\
& y_{i}(t)=y-a \cos \alpha_{i}-b \cos \beta_{i} \\
& \varphi=\arctan \left(l_{1} / l_{2}\right), \quad l_{1}=A_{i} H_{i}, l_{2}=A_{i} T_{i} \\
& \angle H_{i} A_{i} T_{i}=\pi / 2 .
\end{aligned}
$$

Consider the following optimal control problem.

Problem A. It is required to determine the state vector $\mathbf{z}(\mathbf{t})$ of the robot and the vector of the controlling stimuli $\mathbf{u}(t)$ which both satisfy the equations of motion (1), the kinematic constraints $(2),(3)$, the boundary conditions

$$
\begin{aligned}
& \mathbf{z}_{x}(T)=\mathbf{z}_{x}(0), \quad x(T)=x(0)+2 L \\
& \mathbf{z}_{x}=\left\{\dot{x}, y, \dot{y}, \psi, \dot{\psi}, \alpha_{i}, \dot{\alpha}_{i}, \beta_{i}, \dot{\beta}_{i}, \gamma_{i}, \dot{\gamma}_{i}\right\},
\end{aligned}
$$

the restrictions on the phase coordinates and controlling stimuli

$$
\begin{aligned}
& y(t) \geq h, \quad \alpha_{i}(t) \geq \beta_{i}(t), \quad t \in[0, T] \\
& y_{H 1}(t)>0, \quad y_{T 1}(t)>0, \quad t \in\left[t_{3}, T\right] \\
& y_{H 2}(t)>0, \quad y_{T 2}(t)>0, \quad t \in\left[t_{1}, t_{2}\right] \\
& R_{i y}(t) \geq 0, \quad t \in[0, T], \quad i=1,2 \\
& x_{H 1}^{0} \leq x_{R 1}(t) \leq x_{H 1}^{0}+l, \quad t \in\left[0, t_{2}\right] \\
& x_{R 1}(t)=x_{H 1}^{0}+l, \quad t \in\left[t_{2}, t_{3}\right] \\
& x_{R 2}(t)=x_{T 2}, \quad t \in\left[0, t_{1}\right] \\
& x_{H 1}^{0}+L \leq x_{R 2}(t) \leq x_{H 1}^{0}+L+l, \quad t \in\left[t_{2}, T\right] \\
& \gamma_{2}(t)-\beta_{2}(t)-\pi / 2=\Theta_{a 2}(t), t \in[0, T] \\
& \alpha_{2}(t)-\beta_{2}(t)=\Theta_{k 2}(t), \quad t \in[0, T] \\
& \alpha_{i}(t)-\psi(t)=\Theta_{h i}(t), t \in[0, T], \quad i=1,2
\end{aligned}
$$

$$
\begin{array}{ll}
R_{2 x}(t)=R_{2 x}^{e}(t), & t \in\left[0, t_{1}\right] \bigcup\left[t_{2}, t_{3}\right] \\
R_{2 y}(t)=R_{2 y}^{e}(t), & t \in\left[0, t_{1}\right] \bigcup\left[t_{2}, t_{3}\right],
\end{array}
$$

and which minimise the functional $[6,7,9,10]$ :

$E=\frac{1}{2 L} \int_{0}^{T} \sum_{i=1}^{2}\left[q_{i}\left(\dot{\psi}-\dot{\alpha_{i}}\right)|+| u_{i}\left(\dot{\alpha_{i}}-\dot{\beta_{i}}\right) \mid+\right.$

$\left.\left|p_{i}\left(\dot{\beta}_{i}-\dot{\gamma}_{i}\right)\right|\right] d t$

The functions $\Theta_{a 2}, \Theta_{k 2}, \Theta_{h i}, R_{2 x}^{e}, R_{2 y}^{e}$, $(i=1,2)$ are given from the experimental data of human locomotion [8]. The boundary conditions (4) and constraints $(2),(3),(5)$ are imposed on the phase state and controlling stimuli of the BWR with the aim to approach its controlling motion to human gait as close as possible.

The problem of designing the rotational spring-damper actuators of the BWR is formulated as follows.

Problem B. Let $\mu(\mathrm{t})$ be the control torque acting at some joint ( $\mu$-joint) of the leg during the energy-optimal motion of the BWR. It is required to determine the vector $\xi_{\mu}$, which minimise the functional

$$
\Phi_{\mu}=\int_{0}^{T}\left[\mu-w\left(t, \xi_{\mu}\right)\right]^{2} d t
$$

where

$$
\begin{aligned}
& w=\left\{\begin{array}{l}
d_{\mu 1}+c_{\mu 1} \varphi_{\mu}(t)+k_{\mu 1} \dot{\varphi}_{\mu}(t), t \in\left[0, t_{\mu}\right] \\
d_{\mu 2}+c_{\mu 2} \varphi_{\mu}(t)+k_{\mu 2} \dot{\varphi}_{\mu}(t), t \in\left[t_{\mu}, T\right]
\end{array}\right. \\
& \xi_{\mu}=\left\{d_{\mu j}, c_{\mu j}, k_{\mu j}, t_{\mu}, j=1,2\right\} \\
& \mu=\left\{q_{i}^{*}(t), u_{i}^{*}(t), p_{i}^{*}(t), i=1,2\right\} .
\end{aligned}
$$

In formulae (8): $c_{\mu j}, k_{\mu j}$ are the torsional spring and damping coefficients of the actuator acting at the $\mu$-joint, respectively; $\varphi_{\mu}(t), \dot{\varphi}_{\mu}(t)$ 
are the angle between the links connected by the $\mu$-joint and the corresponding angular velocity during the energy-optimal motion of the BWR; $t_{\mu}, 0 \leq t_{\mu} \leq T$ is the time of switching of the actuator's parameters; $d_{\mu j}$ is determined by the free angle of the spring and torsional spring coefficient, $(j=1,2)$.

The function (8) with the vector $\xi_{\mu}$ which minimise the functional (7) will determine the linear viscoelastic actuator of the $\mu$ joint of the BWR.

\section{RESULTS AND DISCUSSION}

The proposed approach for the design of the rotational springdamper actuators based control system of the BWR requires the solution of Problems A and B. The algorithm of the numerical solution of Problem A is developed [10] using the special Fourier and spline approximation of the independent variable functions and inverse dynamics method.

Below all numerical results are presented for the following values of the linear and mass-inertia parameters of the BWR: $m, r, J$ are equal to $46.7 \mathrm{~kg}, 0.39 \mathrm{~m}$ and $7.1 \mathrm{kgm}^{2}$, respectively; $m_{a}, r_{a}, a, J_{a}$ are equal to $7.08 \mathrm{~kg}, 0.16 \mathrm{~m}, 0.41 \mathrm{~m}$ and $0.082 \mathrm{kgm}^{2}$, respectively; $m_{b}, r_{b}, b, J_{b}$ are equal to $5.04 \mathrm{~kg}, 0.203 \mathrm{~m}, 0.5 \mathrm{~m}$ and $0.053 \mathrm{kgm}^{2}$, respectively; $l_{1}=0.05 \mathrm{~m}, l_{2}=0.2 \mathrm{~m}$. These values of the parameters of the BWR correspond to the respective parameters of human body with total mass $M=73.2 \mathrm{~kg}$ and height of $1.76 \mathrm{~m}$ [11].

Some results of the solution of Problem A for the gait with natural cadence ( $T=1.1196 \mathrm{~s}, L=0.755 \mathrm{~m})$ [8] are shown in Fig.2-10 (dashed curves).

Figure 2 shows the way in which the knee angle $\Theta_{k 1}(t)=\alpha_{1}(t)-\beta_{1}(t)$ of the leg of the robot changes in time over a double step for the obtained energetically optimal law of motion.

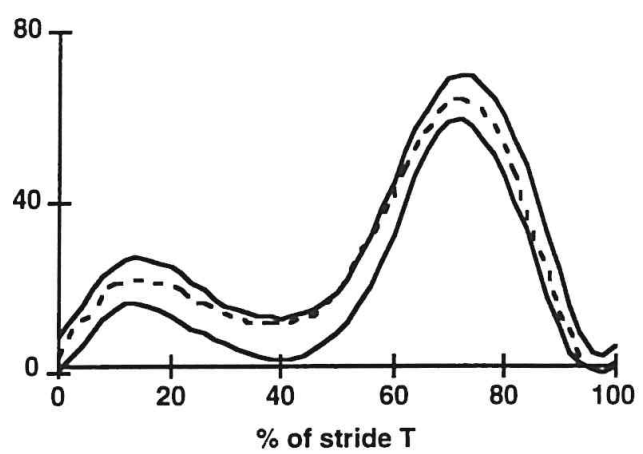

Fig. 2 Knee angle $\Theta_{k 1}(t)$, in degrees

The way in which the specific horizontal component $R_{1 x}(t) / M$ of the support reaction varies (Fig.3, dashed curve) indicates that in each single step the support leg successively executes two functions: deceleration of the robot (time interval in which $R_{1 x}(t) / \mathrm{M}<0$ ) and separation (time interval in which $\left.R_{1 x}(t) / M>0\right)$. The maximum value of the $R_{1 x}(t)$ amounts to $20 \%$ of the entire weight of the robot. The vertical component of the support reaction $\left(R_{l y}(t) / M\right.$, Fig.4, dashed curve) exceeds the weight of the robot by not more then $7 \%$.

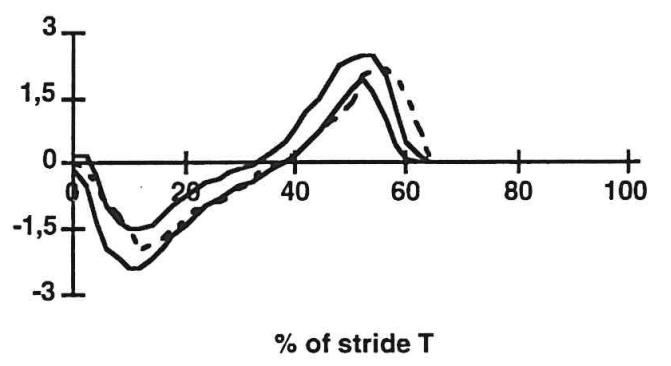

Fig.3 Horizontal force $R_{1 x}(t) / M$, in N/kg 


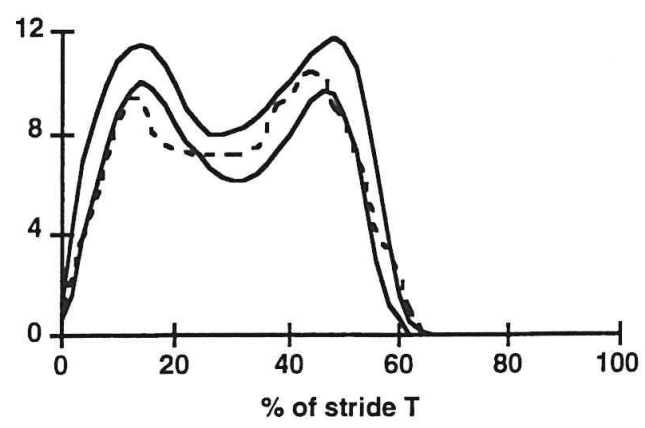

Fig.4 Vertical force $R_{1 y}(t) / M$, in N/kg

For comparison purposes in Fig.2-4 the domains of the values of the respective characteristics obtained by experiments for human normal gait are shown (the domains are bounded by the solid curves) [8].

The analysis of these data and all above mentioned indicate that the kinematic and dynamic characteristics of the obtained energy-optimal law of motion of a BWR are within reasonable proximity to the corresponding characteristics of human gait.

Figures 5-10 show the specific control torques

$$
q_{i}^{*}(t) / M, u_{i}^{*}(t) / M, p_{i}^{*}(t) / M, i=1,2
$$

(dashed curves) acting at the joints of the legs during the energy-optimal law of motion of the robot.

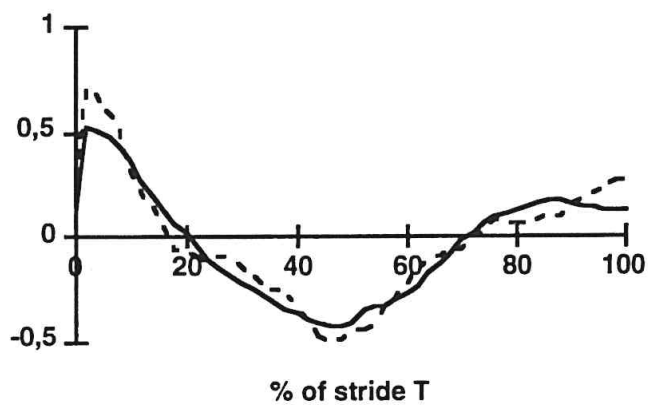

Fig.5 Hip torque $q_{1}^{*}(t) / M$, in $\mathrm{Nm} / \mathrm{kg}$

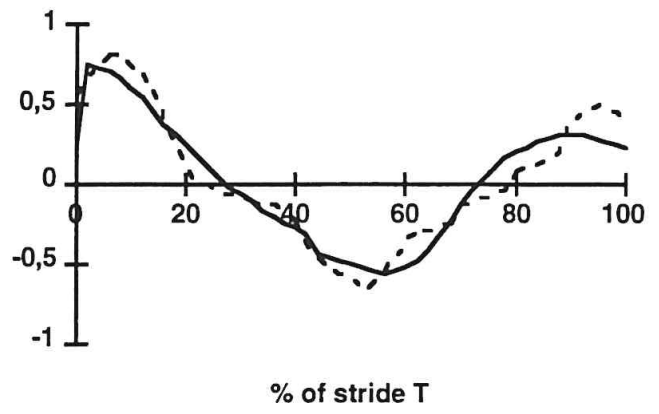

Fig. 6 Hip torque $q_{2}^{*}(t) / M$, in $\mathrm{Nm} / \mathrm{kg}$

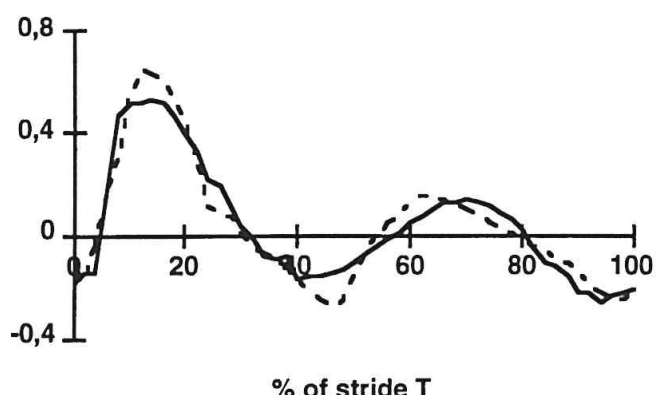

Fig.7 Knee torque $u_{1}^{*}(t) / M$, in $N m / \mathrm{kg}$

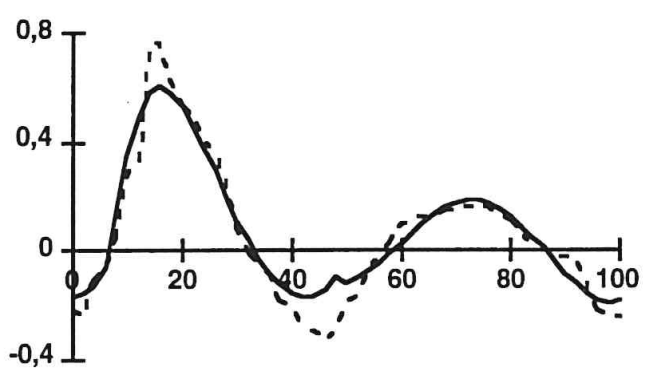

$\%$ of stride T

Fig. 8 Knee torque $u_{2}^{*}(t) / M$, in $N m / k g$

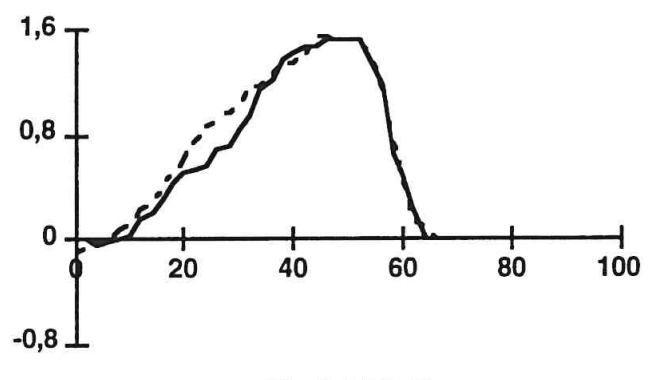

$\%$ of stride $T$

Fig.9 Ankle torque $p_{1}^{*}(t) / M$, in $\mathrm{Nm} / \mathrm{kg}$ 


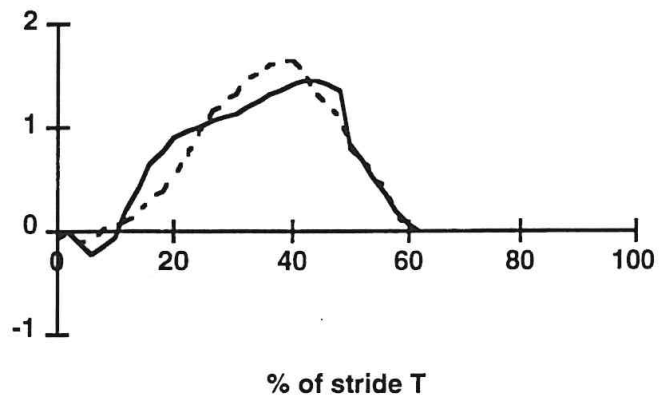

Fig. 10 Ankle torque $p_{2}^{*}(t) / M$, in $\mathrm{Nm} / \mathrm{kg}$

The solution of Problem $B$, that is $\quad \mathrm{the} \quad \mathrm{vectors}$ $\xi_{\mu}=\left\{d_{\mu j}, c_{\mu j}, k_{\mu j}, t_{\mu}, j=1,2\right\}$, for these optimal control torques are determined by data of the Table.

The graphic dependencies of the viscoelastic approximation of the controlled torques for the energyoptimal motion of BWR are depicted in Figures 5-10 (solid curves).

The analysis of these graphic dependencies shows that the control stimuli required for the energyoptimal motion of the BWR can be constructed with sufficient accuracy by the torsional spring-damper actuators. It is also can be noted that these control stimuli quantitatively and qualitatively are within reasonable proximity to the corresponding characteristics of human locomotion [8]. This is additional evidence of fruitfulness of the proposed approach for modelling of bipedal walking.

The analysis of the data of Table shows that within the framework of our assumption the spring coefficients of the designed energyoptimal actuators are one-two order of magnitude larger then the damping coefficients. The stiffness parameters of the actuators of ankles joints of the BWR are the greatest and are approximately 2-3 times greater then those of the actuators of hip joints.

Table

Torsional spring $(\mathrm{Nm} / \mathrm{rad})$ and damping $(\mathrm{Nms} / \mathrm{rad})$ coefficients of the actuators and time $(\% \mathrm{~T})$ of their switching

\begin{tabular}{lcccccc} 
& $q_{1}^{*}(t) / M$ & $q_{2}^{*}(t) / M$ & $u_{1}^{*}(t) / M$ & $u_{2}^{*}(t) / M$ & $p_{1}^{*}(t) / M$ & $p_{2}^{*}(t) / M$ \\
\hdashline$c_{\mu 1}$ & 1.821 & 2.249 & 3.361 & 2.991 & 5.361 & 5.224 \\
$k_{\mu 1}$ & 0.180 & 0.103 & 0.048 & 0.090 & 0.082 & 0.003 \\
$d_{\mu 1}$ & -0.013 & 0.059 & -0.745 & -0.553 & 0.289 & 0.248 \\
$c_{\mu 2}$ & 0.886 & 1.482 & 0.319 & 0.336 & 3.411 & 1.835 \\
$k_{\mu 2}$ & 0.003 & 0.049 & 0.011 & 0.002 & 0.002 & 0.066 \\
$d_{\mu 2}$ & 0 & 0 & 0 & 0 & 0 & 0 \\
$t_{\mu}$ & 48 & 46 & 44 & 46 & 36 & 36 \\
& & & & & &
\end{tabular}

\section{CONCLUSION}

In this paper the analysis of the goal-directed motion of the BWR is based on the solution of energyoptimal control problem for a plane multibody system with nine degrees of freedom. Taking into account the obtained results the following conclusions can be drawn.

The proposed optimisation approach for designing of the rotational spring-damper actuators 
based control system of a BWR is fruitful.

The anthropomorphic energyoptimal motion of the BWR could be generated by the rotational springdamper actuators with one switching for each of their parameters during the double step of the robot.

\section{ACKNOWLEDGEMENTS}

This work was partially supported by The Wenner-Gren Center Foundation and by The Swedish Institute, Stockholm, Sweden.

We are also grateful for the kind hospitality of professors Anders Boström and Peter Olsson at the Department of Mechanics, Chalmers University of Technology, Göteborg, Sweden.

\section{REFERENCES}

1. M. Vukobratovich, Walking Robots and Anthropomorphic Mechanism, Mir Press, Moscow, 1976.

2. V.B. Larin, Control of walking apparatuses, Naukova Dumka, Kiev, 1980.

3. H. Hatze, Neuromusculoskeletal control systems modeling - a critical survey of recent developments, IEEE Transactions on Automatic Control, AC-25 5 (1980) 375-385.

4. A.M. Formal'sky, Displacement of anthropomorphic mechanisms, Nauka, Moskow, 1982.

5. V.V. Beletskii, V.E. Berbyuk and V.A. Samsonov, Parametric optimisation of motion of a bipedal walking robot, J. Mechanics of Solids, 17 (1982) 24-35.
6. V.V. Beletskii, Two-Legged Walking: Model Problems of Dynamics and Control, Nauka, Moscow, 1984.

7. V.E. Berbyuk, Dynamics and Optimisation of Robototechnical Systems, Naukova Dumka, Kiev, 1989.

8. D. Winter, The Biomechanics and Motor Control of Human Gait, University of Waterloo Press, Canada, 1991.

9. V. Berbyuk, Dynamic and optimal control problems for biotechnical systems "Man-Prosthesis". In D.H. van Campen (editor), I U T A M Symposium on Interaction Between Dynamics and Control in Advanced Mechanical Systems, Kluwer Academic Publishers, The Netherlands, (1997) 35-43.

10. V. Berbyuk, G. Krasyuk and N. Nishchenko, Mathematical modelling of the human gait dynamics in sagittal plane, $J$. Matematuchni Metody $i$ FizykoMechanichni Polya, Ukrainian National Academy of Sciences, 404 (1997) 65-76.

11. A. Cappozzo, T. Leo and A. Pedotti, A general computing method for the analysis of human locomotion, J. Biomechanics, 8 (1975) 307-320.

This paper has excepted for presentation at The $6^{\text {th }}$ UK Mechatronics Forum International Conference, 9-11 September 1998, University of Skövde, Sweden and to be published in: PROCEEDINGS OF THE $6^{\text {th }}$ UK MECHATRONICS FORUM INTERNATIONAL CONFERENCE. 